\title{
Metahemoglobinemia por fenazopiridina: reporte de un caso con enfoque diagnóstico
}

\author{
Methemoglobinemia by phenazopyridine: a case report \\ with diagnostic approach
}

Jorge Guillermo Pérez-Tuñón, ${ }^{1}$ Rocío Martiñón-Ríos, ${ }^{2}$ Magaly Figueroa-Rivera ${ }^{3}$

\begin{abstract}
Resumen
INTRODUCCIÓN: La metahemoglobinemia adquirida es un trastorno en el que la hemoglobina es oxidada por un agente externo, impidiendo el transporte y entrega del oxígeno a los tejidos. Uno de los agentes oxidantes es el clorhidrato de fenazopiridina, una anilina con efecto analgésico empleada para tratar el dolor de la vía urinaria baja. CASO CLínICO: Paciente de 12 años, que ingirió $250 \mathrm{mg}$ de amitriptilina, $1.8 \mathrm{~g}$ de fenazopiridina y $14.5 \mathrm{mg}$ de clonazepam en un intento suicida, desarrollando metahemoglobinemia que requirió el empleo de azul de metileno en dos ocasiones.

CONCLUSIONES: La metahemoglobinemia es subdiagnosticada a pesar de poder originar complicaciones graves e, incluso, la muerte. Se diagnostica mediante cooximetría. Cuando ésta no está disponible, además de los datos clínicos, debe evaluarse la brecha de saturación y la sangre color "chocolate".

PALABRAS CLAVE: Metahemoglobinemia; hemoglobina; oxígeno; agentes oxidantes; fenazopiridina; azul de metileno.
\end{abstract}

Abstract

INTRODUCTION: Acquired methemoglobinemia is a disorder in which hemoglobin is oxidized by an external agent, blocking transport and delivery of oxygen to tissues. One of the oxidizing agents is phenazopyridine hydrochloride, an aniline with analgesic effect used to treat urinary tract pain.

CASE REPORT: We present the case of a 12-year-old female patient who swallowed $250 \mathrm{mg}$ of amitriptyline, $1.8 \mathrm{gr}$ of phenazopyridine and $14.5 \mathrm{mg}$ of clonazepam on a suicide attempt, developing metahemoglobinemia that required administration of methylene blue twice.

CONCLUSIONS: Methemoglobinemia is an underdiagnosed condition that can lead to serious complications and even death. It is diagnosed by co-oximetry. When this is not available, in addition to the clinical data, the saturation gap and the presence of "chocolate" blood should be evaluated.

KEYWORDS: Methemoglobinemia; Hemoglobin; Oxygen; Oxidizing agents; phenazopirydine Methylene Blue.

\author{
1 Jefe del Centro Toxicológico. \\ ${ }^{2}$ Adscrita al Centro Toxicológico. \\ ${ }^{3}$ Residente de quinto año de Toxicología \\ Clínica.
}

Hospital Ángeles Lomas, Huixquilucan, Estado de México.

Recibido: 2 de octubre 2017

Aceptado: 21 de agosto 2018

\section{Correspondencia}

Jorge Guillermo Pérez Tuñón

jtunon80@hotmail.com

Este artículo debe citarse como Pérez-Tuñón JG, Martiñón-Ríos R, Figueroa-Rivera M. Metahemoglobinemia por fenazopiridina: reporte de un caso con enfoque diagnóstico. Acta Pediatr Mex. 2018;39(6):355-360.

\section{INTRODUCCIÓN}

La metahemoglobina es un estado patológico de la hemoglobina en el que el hierro que contiene el grupo hemo es oxidado por un agente diferente al oxígeno, permaneciendo en su forma oxidada o férrica $\left(\mathrm{Fe}^{3+}\right)$, en lugar de su forma reducida o ferrosa $\left(\mathrm{Fe}^{2+}\right)$. La hemoglobina sólo puede trasportar oxígeno cuando el átomo de hierro se encuentra en su forma ferrosa, por lo que en estado férrico condiciona estados de hipoxia. 
En condiciones normales existe una concentración tolerable de metahemoglobina en sangre, regulada por la función de los sistemas reductores de los eritrocitos (citocromo-b5 reductasa y $\mathrm{NADPH}^{+}$, principalmente). Su concentración es de entre 1 y $2 \%$ de la hemoglobina total. Cuando la capacidad compensadora de estos sistemas reductores se rebasa, el porcentaje de metahemoglobina se incrementa, lo que provoca una desviación a la izquierda de la curva de disociación de la hemoglobina y disminución en el aporte de oxígeno a los tejidos en una condición denominada metahemoglobinemia. ${ }^{1-3}$

Si bien la metahemoglobinemia puede ser hereditaria, la forma más común es la adquirida y se debe a la exposición a agentes oxidantes: medicamentos, antisépticos, pesticidas, colorantes y fertilizantes, además de otros agentes. ${ }^{3-7}$

\section{Cuadro 1}

Cuadro 1. Agentes metahemoglobinizantes

\begin{tabular}{|c|c|c|}
\hline Acetanilida & Fenacetina & Nitrobenceno \\
\hline Ácido valproico & Fenitoína & Nitrofurantoína \\
\hline Aminofenol & Fenol & Nitroglicerina \\
\hline Anilinas & Flutamida & Nitroprusiato \\
\hline Azul de metileno & Hidroxilamina & Nitritos** \\
\hline Benzocaína & Humo & Nitrofenol \\
\hline Bupivacaína & Lidocaína & Óxido nítrico \\
\hline $\begin{array}{l}\text { Butilnitrilo/isobu- } \\
\text { tilnitrilo }\end{array}$ & Metoclopramida & Óxido nitroso \\
\hline Clorobenceno & Naftaleno & Paraquat \\
\hline Cloratos & $\begin{array}{l}\text { Nitrato de amo- } \\
\text { nio }\end{array}$ & Piperazina \\
\hline Cromatos & Nitrato de sodio & Prilocaína \\
\hline Dimetil sulfóxido & Nitrato de plata & $\begin{array}{l}\text { Quinonas } \\
\text { (cloroquina) }\end{array}$ \\
\hline Dinitrofenol & Nitratos* & Rifampicina \\
\hline Cobre bivalente & Nitrito de amilo & Riluzol \\
\hline Dapsona & Nitrito de isobutil & Toluidina \\
\hline Fenazopiridina & Nitrofenol & Trinitrotolueno \\
\hline
\end{tabular}

*Se encuentran en grandes cantidades en el agua de pozo, fertilizantes y hortalizas regadas con agua tratada.

** Se encuentran en embutidos, quesos y vinos.
Pese a que se desconoce la incidencia precisa de la metahemoglobinemia en el contexto global, en Estados Unidos, durante el 2015, se registró la administración del antídoto azul de metileno a 166 pacientes, de los que solo 31 (18.6\%) tenían 19 años o menos. ${ }^{8}$

El clorhidrato de fenazopiridina es una anilina con efecto analgésico local, que por su elevada eliminación sin cambios en la orina (más de $65 \%$ ) se utiliza con frecuencia para tratar el dolor en pacientes con infecciones urinarias bajas o en quienes se ha realizado algún procedimiento en el aparato urinario. La fracción que es biotransformada en su mayoría lo hace por hidroxilación para formar los metabolitos activos: p-aminofenol, $\mathrm{N}$-acetil-p-aminofenol, anilina y triaminopiridina. Tiene una vida media de eliminación de 7.35 horas y, al igual que con otras anilinas, la fenazopiridina y sus metabolitos se comportan como agentes oxidantes. ${ }^{9-12}$ En Estados Unidos, en 2015, se registraron 1022 exposiciones a fenazopiridina de las que 634 (62\%) correspondieron a pacientes de 19 años o menos, solo se reportaron 65 casos intencionales $(6.3 \%)$ y $6(0.5 \%)$ con potencial riesgo para la vida. ${ }^{8}$

\section{CASO CLÍNICO}

Paciente femenina de 12 años, sin antecedentes de enfermedad crónica o intentos previos de autolisis. Fue llevada a urgencias aproximadamente 1 hora después de ingerir 10 tabletas de amitriptilina de $25 \mathrm{mg}$ (total: $250 \mathrm{mg}$ ), 18 tabletas de fenazopiridina de $100 \mathrm{mg}$ ( $1.8 \mathrm{gr}$ ) y 29 tabletas de clonazepam de $0.5 \mathrm{mg}$ (14.5 mg), con fines autolesivos, refiriéndose que durante su traslado tuvo deterioro del estado de alerta.

A su ingreso se encontró con FC 96 I/m, FR 12 $\mathrm{r} / \mathrm{m}$, TA $116 / 76 \mathrm{mmHg}$, temperatura $36.7^{\circ} \mathrm{C}$, un puntaje de 8 en la escala de coma de Glasgow (O: 2, V: 2, M: 4), con palidez generalizada, 
cianosis distal y saturación de oxígeno al 85\% por oximetría de pulso con $\mathrm{FiO}_{2}$ al $21 \%$. Se indicó tratamiento avanzado de la vía aérea, con secuencia rápida de intubación y se llevó a cabo la descontaminación gástrica mediante lavado. Se obtuvo contenido positivo, con color amarillo-naranja y restos de tabletas en cantidad moderada. Posteriormente se administró carbón activado, que se continuó en dosis múltiples.

Pese al apoyo ventilatorio con aporte de $\mathrm{FiO}_{2}$ al $100 \%$, la paciente persistió con saturación de oxígeno al $86 \%$ y cianosis distal. Por gasometría se reportó con alcalosis respiratoria compensada e hiperoxemia ( $\mathrm{pH}$ 7.43, $\mathrm{PaCO}_{2}$ 26.1, $\mathrm{PaO}_{2} 246$, $\mathrm{HCO}_{3} 20.5, \mathrm{SatO}_{2} 99.1 \%$, Lact 1.8). Se calculó una brecha de saturación entre el reporte gasométrico y la oximetría de pulso de $13.1 \%$, lo que sugirió dishemoglobinemia, que se confirmó por cooximetría, y valor de metahemoglobina en $24 \%$. Por esto se administró una dosis de azul de metileno a $1 \mathrm{mg} / \mathrm{kg}$ y se solicitó un control 2 horas más tarde, que se reportó en $13.5 \%$ con una brecha de saturación de $8 \%$ y disminución de la cianosis; sin embargo, 6 horas más tarde se observó reaparición de la cianosis con brecha de saturación en $14 \%$ y metahemoglobina en $31.5 \%$ por lo que se administró una segunda dosis de azul de metileno a $1 \mathrm{mg} / \mathrm{kg}$, nuevamente con mejoría, con un control de MetHb en $4.8 \%$ a las 3 horas y una brecha de saturación de 2\%, sin nuevos eventos de cianosis o desaturación por oximetría de pulso. Cuadro 2
Durante su evolución clínica la paciente experimentó cambios electrocardiográficos asociados con intoxicación por amitriptilina, se trataron con bicarbonato de sodio y dosis múltiples de carbón activado, sin complicaciones. A dos días de su ingreso se llevó a cabo la extubación programada y, posteriormente, continuó el tratamiento a cargo de paidopsiquiatría y terapia médico familiar. Posterior a su alta hospitalaria, las pruebas de laboratorio a los 7 y 14 días no mostraron evidencia de hemólisis, lesión renal o hepática.

\section{DISCUSIÓN}

Determinar la incidencia de la metahemoglobinemia es aún un reto epidemiológico mundial porque su etiología es diversa; puede originarse por medicamentos, suplementos dietéticos, alimentos y otros productos de consumo cotidiano. A lo anterior se suma el hecho de que no siempre ocurre en el contexto de una intoxicación, pues en sujetos vulnerables puede manifestarse como una reacción adversa a la administración de un medicamento en dosis terapéuticas, lo que también dificulta su clasificación como entidad patológica. Además, en México y otros países de América Latina, otro factor a considerar es la reducida bibliografía científica disponible en idioma español. ${ }^{13}$

En algunas regiones se ha optado por monitorear la administración de azul de metileno como

Cuadro 2. Evolución del caso

\begin{tabular}{|l|c|l|}
\hline Tiempo desde la exposición & $\%$ de metahemoglobina & \multicolumn{1}{c|}{ Tratamiento } \\
\hline 1 hora & $\begin{array}{c}\text { Llegada al hospital } \\
\text { Vía aérea avanzada, soluciones parenterales, descontami- } \\
\text { nación. }\end{array}$ \\
\hline 1.5 horas & 24.7 & Primera dosis de azul de metileno $60 \mathrm{mg}$ IV. \\
\hline 9.5 horas & 13.5 & ----- \\
\hline 12.5 horas & 31.5 & Segunda dosis de azul de metileno $60 \mathrm{mg}$ IV. \\
\hline
\end{tabular}


antídoto específico para establecer una correlación con los casos de metahemoglobinemia que requieren tratamiento; ${ }^{5}$ no obstante, debe tenerse en mente que el azul de metileno tiene indicación en otras enfermedades: hipotensión arterial resistente, síndrome hepato-pulmonar, priapismo, encefalopatía por ifosfamida y en la prevención de adherencias posquirúrgicas, por lo que tal correlación debe tomarse con reserva. ${ }^{3,14}$

Las unidades hospitalarias donde los recursos para la atención de los pacientes son limitados, se carece de cooxímetros. En estas condiciones el médico se ve obligado a considerar mayormente otros elementos clínicos y paraclínicos que contribuyan al diagnóstico y le permitan el tratamiento oportuno, centrando su sospecha clínica en los pacientes expuestos a un agente metahemoglobinizante y en quienes tuvieron cianosis súbita, sin mejoría con el aporte de oxígeno suplementario. En esta comunicación se expone un caso de metahemoglobinemia en la que a la par del análisis por cooximetría, se consideran otros dos elementos de alta sospecha diagnóstica de este padecimiento: brecha de saturación y la detección de sangre "achocolatada", que, pese a no ser equiparables a la cooximetría como prueba diagnóstica, pueden representar los únicos recursos disponibles para considerar la administración de azul de metileno.

Se reportó el caso de una paciente adolescente con metahemoglobinemia tratada con azul de metileno y posterior recaída que también requirió tratamiento. Si bien el criterio general para tratamiento con azul de metileno en pacientes asintomáticos es a partir de $30 \%$ de metahemoglobina, ${ }^{1-3}$ en nuestra paciente se consideró iniciar con un valor de $24 \%$ debido a que se esperaba un comportamiento ascendente de la metahemoglobina por la cantidad de fenazopiridina que ingirió y el poco tiempo que requirió para alcanzar dicha cifra y porque se tenía el riesgo de morbilidad cardiovascular (hipotensión y arritmias) por la coingesta del antidepresivo cíclico. ${ }^{15,16}$ En este sentido, el deterioro del estado de alerta no fue atribuible a la metahemoglobinemia debido a que también había ingerido una benzodiacepina en sobredosis.

Las recaídas en pacientes con metahemoglobinemia posterior a la aplicación de azul de metileno son poco frecuentes y casi siempre obedecen a una vida media prolongada del xenobiótico, a dosis muy elevadas, circulación entero-hepática o entero-entérica, interacciones medicamentosas o condiciones propias del paciente (edad menor a 3 años o mayor a 65 años, desnutrición, insuficiencia renal, anemia, sepsis y acidosis metabólica, entre las más importantes). ${ }^{1,3}$ En este caso, la recaída de la paciente $(31.5 \%$ a las 9.5 horas de la ingesta) quizá se relacionó con que pese a las medidas de descontaminación Ilevadas a cabo, un porcentaje del medicamento permaneció en la luz intestinal y luego se absorbió. Esto representa un fenómeno común si la dosis fue elevada o si la descontaminación gástrica fue tardía. ${ }^{17}$

En relación con la intoxicación por fenazopiridina, son frecuentes la hemólisis y la insuficiencia renal aguda, ${ }^{18-20}$ que no se evidenciaron en nuestra paciente; con menor frecuencia se han reportado rabdomiólisis y hepatotoxicidad, que tampoco se observaron. ${ }^{21-23}$

El diagnóstico confirmatorio se efectuó mediante cooximetría, lo que permitió también evaluar la respuesta al tratamiento; sin embargo, existen otras herramientas útiles cuando no se cuenta con este recurso. Un interrogatorio dirigido y la evaluación clínica minuciosa son complemento fundamental para cualquiera de las técnicas. Para ello puede ser de utilidad correlacionar el grado de afectación clínica con el porcentaje de metahemoglobina (Cuadro 3), aunque las 
Cuadro 3. Porcentaje de metahemoglobina y su correlación clínica

\begin{tabular}{l|l}
$\begin{array}{l}\text { Metahemoglobina } \\
(\%)\end{array}$ & \multicolumn{1}{c}{ Datos clínicos } \\
\hline $0.5-3$ & $\begin{array}{l}\text { Normal, asintomático. } \\
\text { Posiblemente asintomático, palidez } \\
\text { grisácea en pacientes de piel clara, } \\
\text { disnea en pacientes con enfermedad } \\
\text { pulmonar, cardiaca o anemia pre- } \\
\text { existentes. }\end{array}$ \\
\hline $15-15$ & $\begin{array}{l}\text { Cianosis, baja saturación, disnea de } \\
\text { medianos esfuerzos, sangre "acho- } \\
\text { colatada". }\end{array}$ \\
\hline $20-50$ & $\begin{array}{l}\text { Confusión, mareo, fatiga, debilidad, } \\
\text { disnea de pequeños esfuerzos o en } \\
\text { reposo, cefalea, náusea, taquicardia, } \\
\text { síncope y dolor precordial. }\end{array}$ \\
\hline $50-70$ & $\begin{array}{l}\text { Taquipnea, crisis convulsivas, coma, } \\
\text { arritmias y acidosis láctica. }\end{array}$ \\
\hline 70 & $\begin{array}{l}\text { Hipoxia severa: infarto cerebral, mio- } \\
\text { cárdico, intestinal, muerte. }\end{array}$
\end{tabular}

La aparición de los datos clínicos puede ser con valores inferiores de metahemoglobina en pacientes con anemia, insuficiencia cardiaca, enfermedad pulmonar y obesidad mórbida, principalmente.

condiciones previas del paciente como la edad, comorbilidades o su tono de piel pueden alterar esa correlación. ${ }^{3}$

Cuando no es posible obtener la concentración de metahemoglobina por cooximetría, un dato clínico de sospecha para metahemoglobinemia es la brecha de saturación, que también se calculó en este caso. La cuantificación requiere un oxímetro de pulso y un gasómetro, a partir de ello se calcula la diferencia entre la saturación de oxígeno por oximetría de pulso y la saturación de oxígeno por gasometría arterial $\left(\mathrm{SatO}_{2 \mathrm{G}}\right.$ - $\mathrm{SatO}_{2 \mathrm{O}}$ ) que, en condiciones normales, no debe superar $5 \%$, de lo contrario sugiere una alteración conformacional de la hemoglobina o dishemoglobinemia. Se trata de una prueba de baja especificidad porque se modifica con otras formas patológicas de hemoglobina, como la sulfahemoglobina y la carboxihemoglobina; sin embargo, puede sugerir el diagnóstico y la severidad de la metahemoglobinemia si se correlaciona con los datos clínicos y el interrogatorio. ${ }^{24-28}$ Nuestra paciente, al encontrarse con una metahemoglobina de $31 \%$ por cooximetría, se mantenía con una saturación de oxígeno por oximetría de pulso en $85 \%$, mientras que por gasometría arterial se encontraba en $99 \%$, lo que resultó en una brecha de saturación de 14\%, compatible con metahemoglobinemia.

Para los centros que no cuentan con gasómetro o con oxímetro de pulso puede llevarse a cabo la búsqueda de sangre color "chocolate" o "achocolatada". Esta prueba consiste en colocar una gota de sangre arterial del paciente sobre una toalla blanca de papel absorbente o papel filtro, dejarla secar al aire por 60 segundos y observarla, la prueba se considera positiva si al secarse adquiere el característico color marrón chocolate. ${ }^{29}$

Si existe duda respecto de la coloración, la muestra puede compararse con una muestra arterial de otro paciente, siempre que cumpla con los siguientes criterios: 1) El paciente no tenga enfermedad pulmonar, cardiaca o una dishemoglobinemia y 2) su muestra arterial le fue solicitada para evaluar su propia condición física y no solo para obtener un comparativo.

Otra forma de evaluar la coloración de la sangre puede ser mediante la comparación con un gráfico de colores validado para este propósito, Shihana y su grupo proponen una escala de tonos rojo-marrón graduada cada $10 \%$ de metahemoglobina con un rango de 10 a $80 \%$, aunque en ambos casos la identificación de sangre achocolatada se lleva a cabo a simple vista, lo que implica que su positividad dependa del criterio del observador. ${ }^{30}$

\section{CONCLUSIONES}

La metahemoglobinemia es un padecimiento subdiagnosticado, poco común y que puede 
cursar con complicaciones graves e incluso la muerte. La cuantificación del porcentaje de metahemoglobina por cooximetría es la prueba de elección para el diagnóstico. Cuando no se dispone de esta prueba, si está indicado es aceptable iniciar el tratamiento con azul de metileno en pacientes con antecedente de exposición a metahemoglobinizantes, con cianosis súbita sin respuesta a la administración de oxígeno suplementario y que, además, tienen brecha de saturación amplia o sangre "achocolatada".

\section{REFERENCIAS}

1. Ashurst J, Wasson M. Methemoglobinemia: a systematic review of the pathophysiology, detection and treatment. Del Med J. 2011;83(7):203-8.

2. Rehman HU. Methemoglobinemia. West J Med. 2001;175(3):193-6.

3. Price DP, editor. Methemoglobin inducers. New York. Mc Graw-Hill; 2015. 2746 p. (Hoffman RS, Howland MA, Levin NA, editores. Goldfrank's Toxicologic emergencies;127).

4. Wright OR, Lewander JW. Woolf DA. Methemoglobinemia: Etiology, pharmacology and clinical management. Ann Emerg Med. 1999;34(5):646-56.

5. Oz M, et al. Cellular and molecular actions of methylene blue in the nervous system. Med Res Rev. 2011;31(1):93117. doi: 10.1002/med.20177.

6. Alanazi MQ. Drugs may be induced methemoglobinemia. J Hematol Thrombo Dis. 2017;5(3):1-5. doi: 10.4172/23298790.1000270

7. do Nascimento TS, Pereira ROL, Mello HLD, Costa J. Metahemoglobinemia: del diagnóstico al tratamiento. Rev Bras Anestesiol. 2008;58(6):651-64.

8. Mowry JB, et al. Annual Report of the American Association of Poison Control Centers' National Poison Data System (NPDS): 33rd Annual Report. Clin Toxicol. 2016;54(10):9241109. doi: 10.3109/15563650.2015.1102927.

9. Holmes I, et al. Acute Renal Failure and Jaundice without Methemoglobinemia in a Patient with Phenazopyridine Overdose: Case Report and Review of the Literature. Case Rep Nephrol. 2014. Article ID 845372. http://dx.doi. org/10.1155/2014/845372.

10. Gupta OP, Aggarwal KK. Role of phenazopyridine in urinary tract infections. Indian J Clin Pract. 2012;22(9):437-41.

11. Williamson K, Htet N, Nanini S. Methemoglobinemia from prolonged therapeutic use of phenazopyridine: Ann Clin Case Rep. 2016 Aug;1:1085.

12. Gold NA, Bithoney WG. Methemoglobinemia due to ingestion of at most three pills of pyridium in a-2 years-old: case report and review. J Emerg Med. 2003;25(2):143-8.
13. Zyoud $\mathrm{SH}$, et al. Global methaemoglobinemia research output (1940-2013): a bibliometric analysis. Springer Plus 2015;(4):6-26. doi: 10.1186/s40064-015-1431-7.

14. Miclescu A, Wiklund L. Methylene blue, an old drug with new indications? J Anest Terap Int. 2010;(17):35-41.

15. Verbree FC, et al. Tricyclic antidepressant poisoning: cardiovascular and neurological toxicity. Neth J Crit Care. 2016;24(2):16-20.

16. Kerr GW, et al. Tricyclic antidepressant overdose: a review. Emerg Med J. 2001 Jul; 18(4): 236-41. doi: 10.1136/ emj.18.4.236.

17. Christensen $\mathrm{CM}$, et al. Protacted methemoglobinemia after phenazopyridine overdose in an infant. J Clin Pharmacol. 1996;36(2):112-6.

18. Nathan DM, et al. Acute methemoglobinemia and hemolytic anemia with phenazopyridine: possible relation to acute renal failure. Arch Intern Med. 1977;137(11):1636-8.

19. Zimmerman RC, et al. Methemoglobinemia from overdose of phenazopyridine Hydrochloride. Ann Emerg Med. 1980 Mar;9(3):147-9.

20. Onder AM, et al. Acute renal failure due to phenazopyridine (Pyridium) overdose: case report and review of the literature. Pediatr Nephrol. 2006; 21(11): 1760-4.

21. Gavish D, et al. Methemoglobinemia, muscle damage and renal failure complicating phenazopyridine overdose. Isr J Med Sci. 1986 Jan;22(1):45-7.

22. Cohen BL, Bovasso GJ. Acquired methemoglobinemia and hemolytic anemia following excessive Pyridium (phenazopyridine hydrochloride) ingestion. Clin Pediatr.1971;10(9):537-40.

23. Holmes I, et al. Acute renal failure and jaundice without methemoglobinemia in a patient with phenazopyridine overdose: case report and review of the literature. Case Rep Nephrol. doi: 10.1155/2014/845372. Epub 2014 Mar 5.

24. Gupta PM, et al. Benzocaine-induced methemoglobinemia. South Med J. 2000;93(1):83-6.

25. Huang $A$, et al Methemoglobinemia following unintentional ingestion of sodium nitrite. Morbidity and Mortality Weekly Report (MMWR). CDC. 2002;51(29): 639-42.

26. Rausch-Madison S, et al. Methodologic problems encountered with cooximetry in methemoglobinemia. Am J Med Sci. 1997 Sep;314(3):203-6.

27. Annabu EH, et al Severe methemoglobinemia detected by pulse oximetry. Anesth Analg. 2009;108(3):898-9.doi: 10.1213/ane.0b013e318172af73.

28. Haymond $\mathrm{S}$, et al. Laboratory assessment of oxygenation in methemoglobinemia. Clin Chem. 2005;51(2): 434-44.

29. Henreting FM, et al. Interpretation of color change in blood with varying degree of methemoglobinemia. J Toxicol Clin Toxicol. 1988;26(5-6):293- 301

30. Shihana $F$, et al. A simple quantitative bedside test to determine methemoglobin. Ann Emerg Med. 2010;55(2):184-9. doi: 10.1016/j.annemergmed.2009.07.022. 